\title{
Clinical, virological, and biological parameters associated with outcomes of Ebola virus infection in Macenta, Guinea
}

Marie-Astrid Vernet, ${ }^{1}$ Stéphanie Reynard, ${ }^{2,3}$ Alexandra Fizet, ${ }^{2,3}$ Justine Schaeffer, ${ }^{2,3}$ Delphine Pannetier, ${ }^{4}$ Jeremie Guedj, ${ }^{5,6}$ Max Rives, ${ }^{7}$ Nadia Georges, ${ }^{7}$ Nathalie Garcia-Bonnet, ${ }^{7}$ Aboubacar I. Sylla, ${ }^{8}$ Péma Grovogui, ${ }^{8}$ Jean-Yves Kerherve, ${ }^{7}$ Christophe Savio, ${ }^{7}$ Sylvie Savio-Coste, ${ }^{7}$ Marie-Laure de Séverac, ${ }^{7}$ Philippe Zloczewski, ${ }^{7}$ Sandrine Linares, ${ }^{7}$ Souley Harouna, ${ }^{9}$ Bing M'Lebing Abdoul, ${ }^{9}$ Frederic Petitjean, ${ }^{9}$ Nenefing Samake, ${ }^{9}$ Susan Shepherd, ${ }^{9}$ Moumouni Kinda, ${ }^{9}$ Fara Roger Koundouno, ${ }^{9}$ Ludovic Joxe, ${ }^{9}$ Mathieu Mateo, ${ }^{2,3}$ Patrick Lecine, ${ }^{10}$ Audrey Page, ${ }^{2,3}$ Tang Maleki Tchamdja, ${ }^{7}$ Matthieu Schoenhals, ${ }^{1}$ Solenne Barbe, ${ }^{9}$ Bernard Simon, ${ }^{11}$ Tuan Tran-Minh, ${ }^{11}$ Christophe Longuet, ${ }^{12}$ François L'Hériteau, ${ }^{13}$ and Sylvain Baize ${ }^{2,3}$

'Centre Pasteur du Cameroun, Yaounde, Cameroon. 'Unité de Biologie des Infections Virales Emergentes, Institut Pasteur, Lyon, France. ${ }^{3}$ Centre International de Recherche en Infectiologie, Université de Lyon, INSERM, U1111, Ecole Normale Supérieure de Lyon, Université Lyon 1, CNRS UMR5308, Lyon, France. 'Laboratoire P4 Jean Mérieux - INSERM (US003), Lyon, France. IINSERM, Infection, Antimicrobials, Modelling, Evolution, UMR 1137, Université Paris Diderot, Paris, France. ${ }^{6}$ Assistance Publique-Hôpitaux de Paris, Hôpital Bichat Claude Bernard, Paris, France. ${ }^{7}$ Etablissement de Préparation et de Réponse aux Urgences Sanitaires, Ministère de la Santé, Paris, France. ${ }^{8}$ Ministère de la Santé, Conakry, République de Guinée. ${ }^{9}$ Alliance for International Medical Action, Montreuil, France. ${ }^{10}$ Bioaster, Lyon, France. ${ }^{11}$ Croix-Rouge Française, Paris, France. ${ }^{12}$ Fondation Mérieux, Lyon, France. ${ }^{13} \mathrm{CClin}$ Paris-Nord, Paris, France.

Role of funding source: The French Ministry of Foreign Affairs, the Agence Française de Développement, and the Institut Pasteur have funded the equipment and operating costs of the laboratory.

Authorship note: M.A. Vernet, $S$. Reynard, and A. Fizet contributed equally to this work.

Conflict of interest: The authors have declared that no conflict of interest exists.

Submitted: May 31, 2016 Accepted: February 8, 2017 Published: March 23, 2017

Reference information: JCI Insight. 2017;2(6):e88864. https://doi.org/10.1172/jici. insight.88864.
BACKGROUND. The pathogenesis of Ebola virus (EBOV) disease (EVD) is poorly characterized. The establishment of well-equipped diagnostic laboratories close to Ebola treatment centers (ETCs) has made it possible to obtain relevant virological and biological data during the course of EVD and to assess their association with the clinical course and different outcomes of the disease.

METHODS. We were responsible for diagnosing EBOV infection in patients admitted to two ETCs in forested areas of Guinea. The pattern of clinical signs was recorded, and an etiological diagnosis was established by RT-PCR for EBOV infection or a rapid test for malaria and typhoid fever. Biochemical analyses were also performed.

RESULTS. We handled samples from 168 patients between November 29, 2014, and January 31, 2015; 97 patients were found to be infected with EBOV, with Plasmodium falciparum coinfection in $18 \%$. Overall mortality for EVD cases was $58 \%$, rising to $86 \%$ if $P$. falciparum was also present. Viral load was higher in fatal cases of EVD than in survivors, and fatal cases were associated with higher aspartate aminotransferase (AST) and alanine aminotransferase (ALT), C-reactive protein (CRP), and IL-6 levels. Furthermore, regardless of outcome, EVD was characterized by higher creatine kinase (CPK), amylase, and creatinine levels than in febrile patients without EVD, with higher blood urea nitrogen (BUN) levels in fatal cases of EVD only.

CONCLUSION. These findings suggest that a high viral load at admission is a marker of poor EVD prognosis. In addition, high AST, ALT, CRP, and IL-6 levels are associated with a fatal outcome of EVD. Damage to the liver and other tissues, with massive rhabdomyolysis and, probably, acute pancreatitis, is associated with EVD and correlated with disease severity. Finally, biochemical analyses provide substantial added value at ETCs, making it possible to improve supportive rehydration and symptomatic care for patients.

FUNDING. The French Ministry of Foreign Affairs, the Agence Française de Développement, and Institut Pasteur. 
Patients from Macenta and Nzerekore ETC having had samples analyzed by the Pasteur Laboratory between Nov 29, 2014 and Jan 31, 2015 ( $\mathbf{n = 1 8 3 )}$

\begin{tabular}{|} 
Patients excluded $(n=15)$ \\
Pregnant woman $(n=1)$ \\
Age $<1$ year $(n=7)$ \\
Type of sample $(n=6)$ \\
Unknown outcome $(n=1)$
\end{tabular}
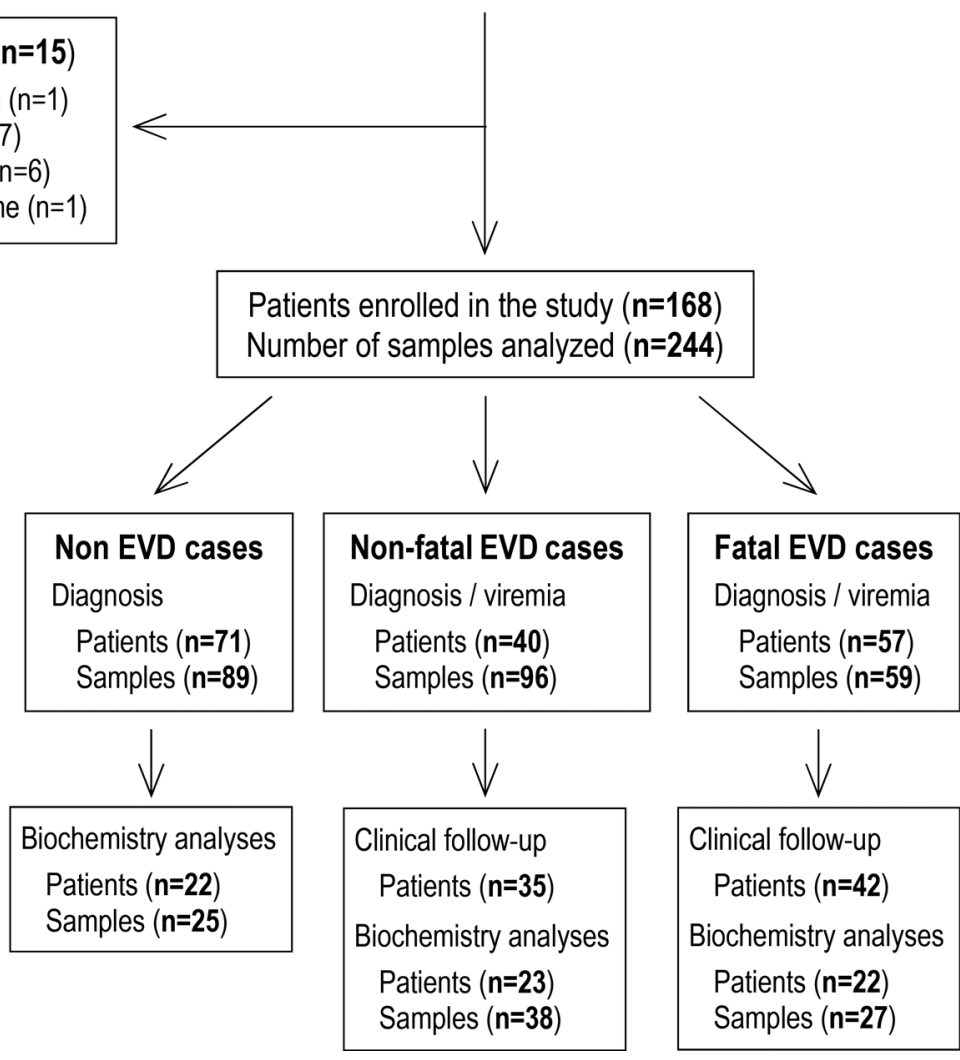

Figure 1. Consort diagram of the study.

\section{Introduction}

The most severe recorded Ebola virus (EBOV) disease (EVD) outbreak ravaged West Africa, mainly Guinea, Liberia, and Sierra Leone, in 2014 and 2015 (1). Previous outbreaks of filovirus disease in Central Africa affected only a small number of people in a limited area for only a few months, but the unusually large extent of this outbreak has required extraordinary resources for its control (2). In September 2014, in the face of increasing numbers of cases in forested areas of Guinea, several Ebola treatment centers (ETCs) were established as a matter of urgency. One of these centers was set up in Macenta, a town with 45,000 inhabitants located in south-east Guinea, close to the borders with Sierra Leone and Liberia. The ETC was funded by the French Ministry of Foreign Affairs, set up by Médecins Sans Frontières, and operated by the French Red Cross, with human resources support from the Etablissement de Préparation et de Réponse aux Urgences Sanitaires (French Ministry of Health). Institut Pasteur set up and ran a labora-

tory close to the ETC to provide diagnostic capacity in the vicinity. The Pasteur Laboratory also dealt with the samples from the ETC at Nzerekore, which was operated by the Alliance for International Medical Action, until the opening of a laboratory associated with this ETC. A high turnover of technical staff was required to operate the laboratory over time, and the teams often lacked experience in handling risk group 4 agents. We therefore decided to provide the laboratory staff with a very high level of biosafety, similar to that used in industrialized countries for the handling of such viruses. The laboratory was set up to provide physicians with facilities for Ebola diagnosis, differential diagnosis for other frequent diseases (malaria and typhoid fever), and the testing of essential biological parameters as well as to improve the supportive rehydration care of patients. Indeed, in the absence of effective specific treatment, symptomatic care was essential, and the higher survival rates observed for repatriated patients receiving intensive care in Northern countries demonstrated the crucial need to improve the standard of care in field facilities. Knowledge about the patterns of change in biological parameters during human EBOV infection was quite limited, but substantial progress has resulted from the last EBOV outbreak. Data for Sudan virus-infected (SUDV-infected) patients demonstrated liver and tissue injury, together with renal failure and possible acute pancreatitis (3). More recent reports of biological follow-up for isolated patients or cohorts have confirmed these results and suggested that rhabdomyolysis is a common features in EVD (4-8). We report here the clinical, virological, and biological parameters recorded for a cohort of EBOV-infected patients hospitalized in the Macenta and Nzerekore ETCs between December 2014 and January 2015 who did not receive any experimental EBOV-specific therapy.

\section{Results}

Clinical description. From November 29, 2014, to January 31, 2015, 183 patients with symptoms consistent with the WHO definition of suspected EVD who were admitted to one of the two ETCs had their samples sent to the laboratory to be tested for diagnostic purposes. Among them, 168 patients were included in the study according 


\begin{tabular}{|c|c|c|c|c|c|c|c|c|c|}
\hline \multirow[b]{2}{*}{ Sex } & \multirow[t]{2}{*}{$n$} & \multicolumn{2}{|c|}{ Death } & \multicolumn{3}{|c|}{ Univariable } & \multicolumn{3}{|c|}{ Multivariable } \\
\hline & & $n$ & $(\%)$ & OR & {$[95 \% \mathrm{Cl}]$} & $P$ & $\mathrm{aOR}$ & {$[95 \% \mathrm{Cl}]$} & $P$ \\
\hline Male & 35 & 18 & (51) & 0.6 & [0.3-1.4] & 0.3 & & & \\
\hline Female & 62 & 39 & (63) & & & & & & \\
\hline \multicolumn{10}{|c|}{$\begin{array}{l}\text { Time between symptom } \\
\text { onset and admission }\end{array}$} \\
\hline$\leq 3$ & 23 & 11 & (48) & 0.3 & [0.1-0.9] & 0.04 & 0.5 & [0.08-3] & 0.4 \\
\hline$<25,000$ & 41 & 16 & (39) & 0.02 & [0.001-0.11] & 0.0002 & 0.07 & [0.003-0.5] & 0.02 \\
\hline$\geq 25,000$ & 33 & 32 & (97) & 1 & & & & & \\
\hline \multicolumn{10}{|l|}{ Baseline titers (log) } \\
\hline$<6$ & 28 & 13 & (46) & 0.07 & [0.003-0.4] & 0.01 & 0.1 & [0.006-1.2] & 0.1 \\
\hline$\geq 6$ & 14 & 13 & (93) & 1 & & & & & \\
\hline $0-9$ & 11 & 10 & (91) & 7 & [0.9-57] & 0.08 & & & \\
\hline $10-19$ & 19 & 9 & (47) & 0.6 & {$[0.2-1.7]$} & 0.5 & & & \\
\hline $20-44$ & 45 & 22 & (49) & 0.7 & [0.3-1.4] & 0.4 & & & \\
\hline$\geq 45$ & 22 & 16 & (73) & 1.9 & {$[0.7-5.2]$} & 0.3 & & & \\
\hline \multicolumn{10}{|c|}{ Presence of $P$. falciparum } \\
\hline Yes & 14 & 12 & (86) & 4 & {$[0.9-27.2]$} & 0.09 & & & \\
\hline No & 60 & 36 & (60) & & & & & & \\
\hline \multicolumn{10}{|c|}{ Ebola treatment center } \\
\hline Nzerekore & 29 & 19 & (65) & 1.0 & [0.4-2.8] & 0.9 & & & \\
\hline Macenta & 45 & 29 & (64) & 1 & & & & & \\
\hline
\end{tabular}

All patients were included for univariate analysis. The age and the delay of 2 and 1 patients, respectively, were missing, and the median value was used. Baseline titers, delay, viremia, and IL-6 were dichotomized using the cutoff maximizing the Youden's index. Age groups were categorized as follows: age category 1: less than 9 years; age category 2: ranging from 10 to 19; age category 3: ranging from 20 to 44; and age category 4: over 44 years old. We chose to be conservative, and only variables with a $P$ value less than 0.05 were included in multivariate analysis. Because IL- 6 and titers were missing in several patients, we checked that the conclusions of the multivariable analysis were unchanged by running two separate analyses where these variables were not included. OR, odds ratio; aOR, adjusted odds ratio.

to our criteria (Figure 1). During this time frame, the laboratory also received samples from 30 patients from places other than the two ETCs, and these were excluded from the study. Testing confirmed EBOV infection in $58 \%(n=97)$ of these patients (mean age: 30.1 years, range: $1-68$ years), and the mortality rate for these cases was $59 \%$ (Table 1). The mortality rate was similar between both ETCs. Although a lower mortality rate has been observed for patients admitted within 3 days after the onset of symptoms, this difference was not significant using multivariate analysis (Table 1). The mean age of the patients was independent of disease outcome, although mortality tended to be higher (though the differences were not significant) in patients under the age of 10 years (91\%) or over the age of 45 years $(73 \%)$. No evidence for recent typhoid fever $\left(\operatorname{IgM}{ }^{+}\right)$was found in any patient. In contrast, we found that $19 \%(n=26)$ of the patients admitted to the ETCs were positive for Plasmodium falciparum. There was no difference in the proportion of $P$. falciparum-positive patients between EBOV-infected and control patients. Coinfection with $P$. falciparum and EBOV seems to be associated with poor prognosis, as a fatal outcome was observed in $86 \%$ of coinfected patients, whereas the mortality rate among $P$. falciparum-negative EBOV-infected patients was $60 \%$. This difference was not significant $(P=0.12)$, but the observed trend suggests that coinfection with $P$. falciparum may increase mortality in patients with EVD. However, of the $P$. falciparumEBOV-positive patients, 7 of 12 were under 10 or above 45 years old, probably accounting for the dramatic mortality observed at these ages. Rather than presenting the clinical signs observed in patients at admission, we have chosen to report the symptoms experienced throughout the course of the disease. Indeed, as the period between the onset of symptoms and admission was highly variable (mean: 4.6 days, range: $<1-14$ days), a comparison 


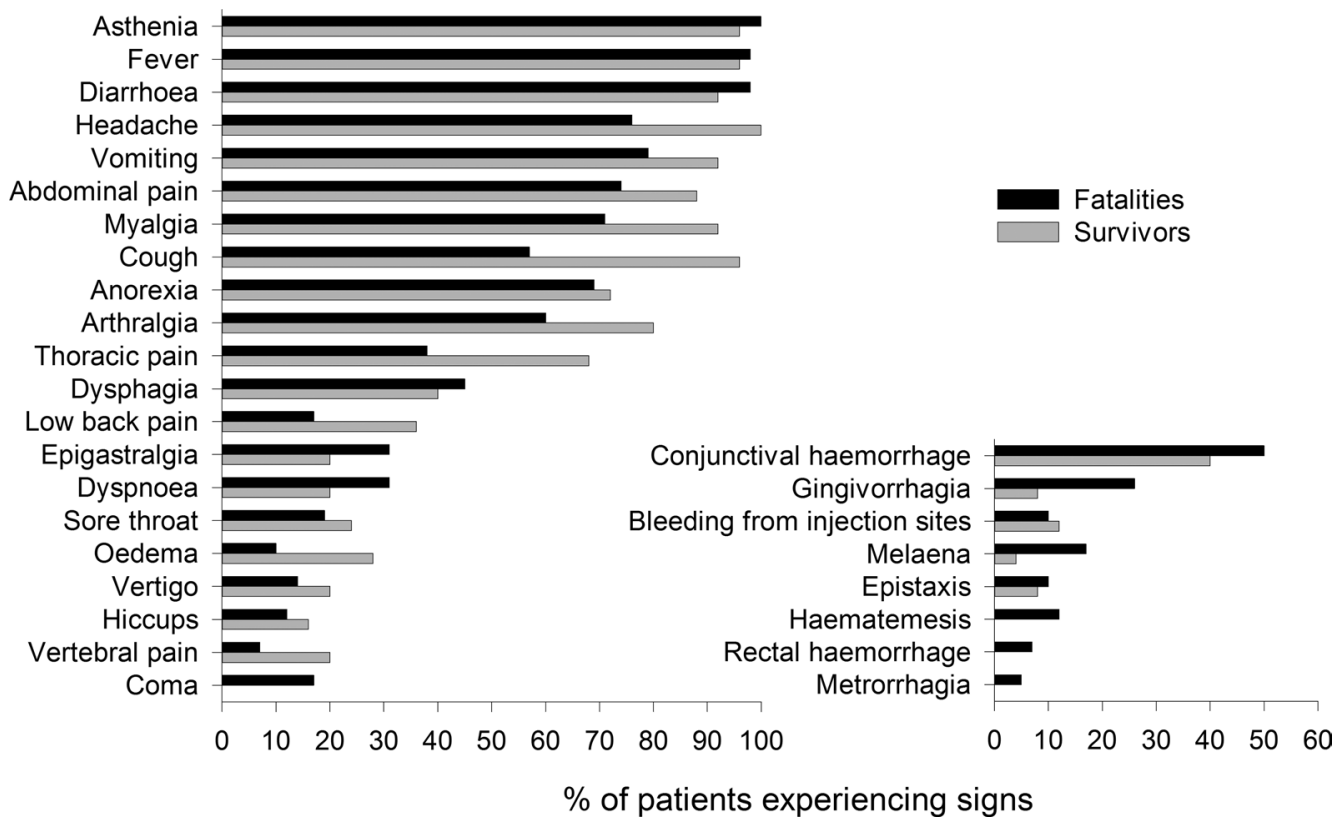

Figure 2. Percentage of Ebola virus disease patients with a fatal $(n=42)$ or nonfatal $(n=35)$ outcome experiencing the various symptoms during the course of the disease.

of clinical presentation at admission between patients would not provide relevant information for this study. Almost all patients for whom we obtained complete clinical follow-up information displayed fever, asthenia, and diarrhea (Figure 2). Fever and asthenia were observed in almost all patients at admission, whereas diarrhea occurred later in the clinical course of the disease. Headache, vomiting, abdominal pain, myalgia, cough, anorexia,

arthralgia, and thoracic pain were observed in most patients. With the exception of anorexia, all these symptoms were reported more frequently in survivors than in fatal cases. But patients with severe illness were very often unable to complain of symptoms such as headache, myalgia, and arthralgia. Moreover, patients with symptoms associated with dehydration or low food and drink intake (diarrhea, vomiting, nausea, hemorrhage) may be less inclined to complain of severe clinical signs, such as headaches, myalgias, and arthralgias, leading to a bias in the clinical description. Other signs, such as dysphagia, low back pain, epigastralgia, dyspnea, sore throat, edema, vertigo, hiccups, vertebral pain, and coma, were less common. Finally, conjunctival hemorrhages were reported in about half of the patients in both the survivor and fatal case groups, whereas other hemorrhagic signs were observed in $5 \%-20 \%$ of fatal cases and $0 \%-10 \%$ of survivors.

Time course of viremia in patients. Viral load was high at admission in all patients, regardless of outcome, even for patients admitted 1 day after disease onset (Figure 3). Viral RNA load at admission was significantly higher in fatal cases than in survivors, confirming that the intensity of viremia at admission could be used as a prognostic marker, and was correlated with disease severity (Table 1). EBOV RNA levels remained high throughout the course of the disease in fatal cases, whereas viremia decreased in survivors about 1 week after the onset of symptoms, with the virus becoming undetectable about 3 weeks after disease onset. However, although viral infectious titers at admission were higher in fatal cases than in survivors, this difference was not significant (Table 1).

Figure 3. Viral load in EBOV-infected patients. (A) Viral RNA load, quantified by real-time RT-PCR, is presented as the median $\pm 95 \% \mathrm{Cl}$ for samples obtained at different times after disease onset in fatal (dark gray squares) and nonfatal (light gray squares) Ebola virus disease (EVD) patients. Individual values are also presented for samples obtained in fatal (red circles) and nonfatal (green circles) EVD patients. Values are expressed in arbitrary units (AU), calculated as follows: $\mathrm{AU}=2^{(34-\text { cycle threshold) }}$, with a limit of detection for the assay corresponding to cycle threshold $(\mathrm{Ct})=34$. The number of samples used to calculate the different median values is indicated for fatal (top line) and nonfatal (bottom line) patients. (B) Infectious viral titers measured at admission are presented with individual values and box plot for fatally infected (Fatal., $n=26$ ) or surviving (Surv., $n=16$ ) patients. Only samples with a $\mathrm{Ct}$ $<28$ were titrated.
A

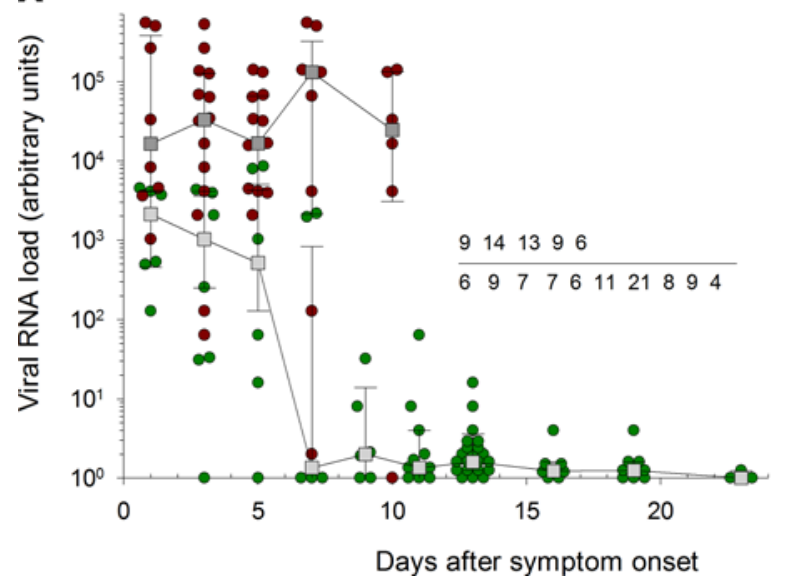

B

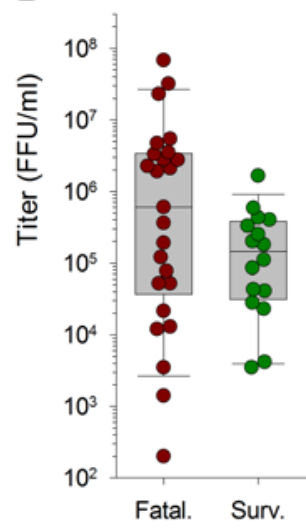


A
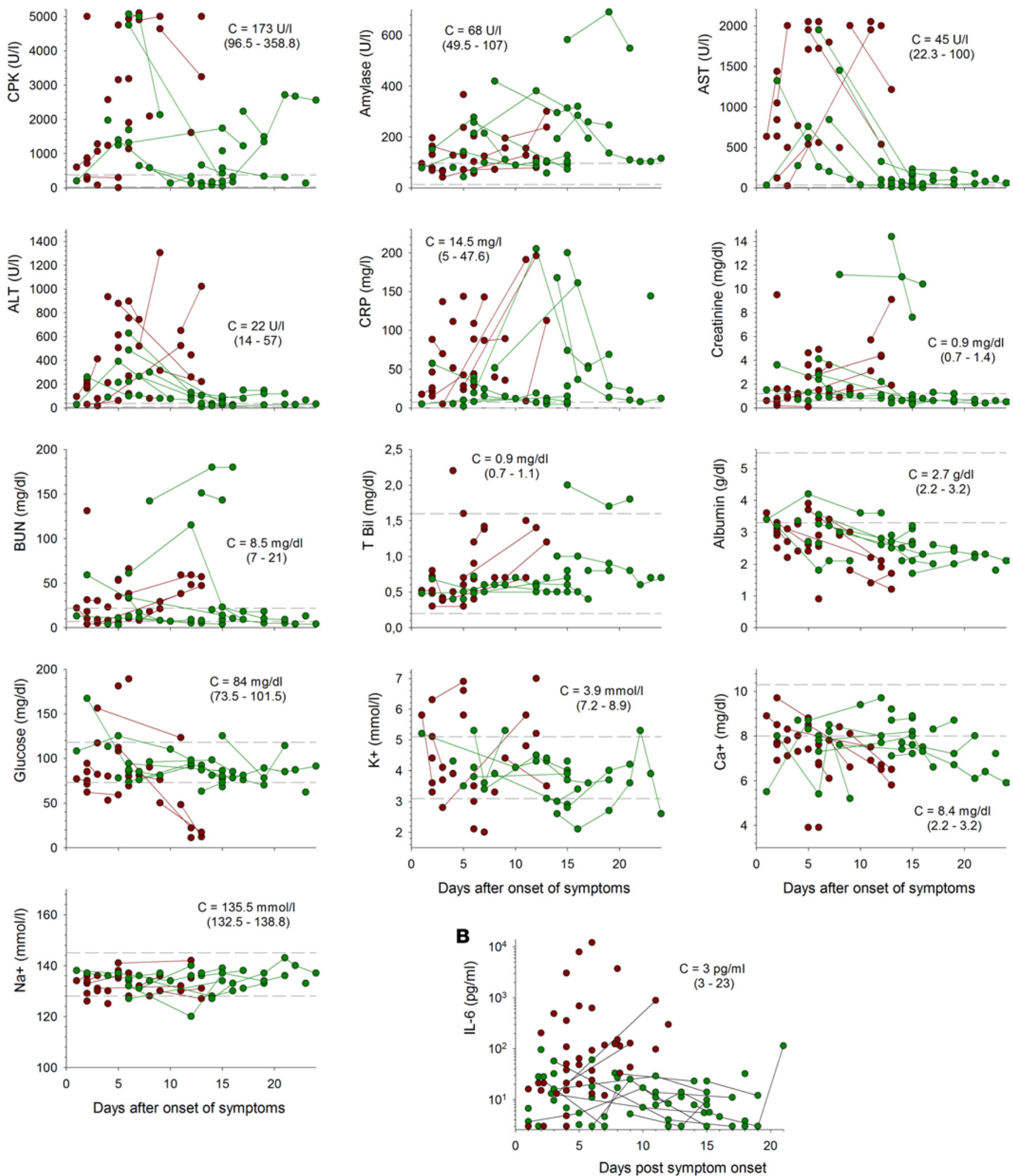

Figure 4. Changes in biological parameters during EVD. (A) Levels of creatine kinase (CPK), amylase, aspartate aminotransferase (AST), alanine aminotransferase (ALT), C-reactive protein (CRP), creatinine, blood urea nitrogen (BUN), total bilirubin (T Bil), albumin, glucose, potassium (K+), corrected calcium $\left(\mathrm{Ca}^{2+}\right)$, and sodium $\left(\mathrm{Na}^{+}\right)$in plasma from fatal (red circles) and nonfatal (green circles) Ebola virus disease (EVD) patients are presented according to the number of days after disease onset. Samples coming from the same patients are linked by a line. Thirty samples from fatal EVD patients are presented, except for AST $(n=25)$, CPK $(n=26), \mathrm{K}^{+}(n=24)$, albumin $(n=29)$, CRP $(n=28)$, and T Bil $(n=27)$. Forty samples from nonfatal EVD patients are presented, except for AST, BUN, CRP, and T Bil $(n=39)$; CPK $(n=38)$; and $\mathrm{K}^{+}(n=37)$. The median $\pm 95 \% \mathrm{Cl}(\mathrm{C})$ of individual values for febrile 
patients without EVD $(n=27)$ is presented. The dotted lines indicate the limits of the range of normal values, as indicated by the assay manufacturer. (B) Plasma IL-6 concentrations for fatal (red circles, $n=43$ ) and nonfatal (green circles, $n=56$ ) EVD cases are shown. Samples coming from the same patients are linked by a line. The median $\pm 95 \% \mathrm{Cl}$ of individual values for febrile patients without EVD $(n=15)$ is presented.

Changes in biological parameters during EVD. Most patients had high levels of creatine kinase (CPK) (Figure 4A and Supplemental Figure 1; supplemental material available online with this article; https://doi.org/10.1172/ jci.insight.88864DS1). These levels increased until death in the fatal cases, whereas, in survivors, they peaked a few days after disease onset but remained high in some patients until recovery. Plasma amylase concentrations were also high in most patients, regardless of outcome, and remained high until the virus was no longer detectable in survivors. Very high levels of aspartate aminotransferase (AST) and alanine aminotransferase (ALT) were observed during the course of the disease. This increase was transient, with the levels of these enzymes decreasing about 1 week after the onset of symptoms, in survivors, whereas they continued to increase relentlessly until death in the fatal cases. Furthermore, a high AST/ALT ratio was typically observed in patients with EVD (mean: $3.93 \pm 0.28$ among viremic patients, $n=41$ ). Levels of C-reactive protein (CRP) were also high during the symptomatic phase, particularly in fatal cases, and these levels seemed to increase earlier in fatal cases than in survivors. Creatinine concentrations were abnormally high during the first half of the symptomatic period in most survivors and were high until death in the fatal cases. Uremia was detected in some survivors and fatal cases, whereas bilirubin levels remained in the normal range in most patients. Albumin concentrations decreased during the course of the disease in both fatal cases and survivors. Glycemia was unaltered in nonfatal infections but decreased steadily until death in fatal cases, with severe hypoglycemia observed in the terminal stages. $\mathrm{K}^{+}$concentrations remained within the normal range in most patients, with only a minority of patients experiencing hypokalemia. This electrolyte imbalance tended to be observed late in the course of the disease in survivors and was not correlated with a poor outcome. Hypocalcemia was not a common feature in EBOV infection, as only a minority of patients had $\mathrm{Ca}^{2+}$ concentrations below the normal range. Finally, $\mathrm{Na}^{+}$levels remained normal throughout the course of the disease in all patients.

Principal component analysis was used to summarize the information represented by the 13 different biological parameters studied in three distinct populations: EVD survivors during acute disease (from 1 to 13 days after onset of symptoms), EVD survivors during early recovery (from 13 to 24 days after disease onset), and fatal EVD cases. The variables driving the transition among these populations, which are, thus, linked to prognosis, are represented as arrows in Figure 5A. The length of these arrows is correlated to the strength of the variable in the transition of one state to another. Contributions of these factors to the first 2 dimensions are represented in Figure 5B and have a cumulative percentage of explained variance of $36.9 \%$. Variables most linked to the transition to fatal cases and to early recovery in survivors are increased blood urea nitrogen, creatinine, and CRP and decreased $\mathrm{Ca}^{+}$and albumin, major contributors of the first dimension. Increase in levels of AST, ALT, CPK, and $\mathrm{K}^{+}$, major contributors of the second dimension, drive, most importantly, the transition to fatal cases only.

Finally, IL-6 levels were moderately increased in the survivors but were nevertheless higher than in negative controls (Figure 4B and Supplemental Figure 1). By contrast, in fatal cases, IL-6 levels were high from 3 to 4 days after symptom onset and remained so until death, with a maximum 5 to 6 days after disease onset. These levels were higher than those observed in negative controls and survivors at 5 to 6 days after the appearance of clinical signs.

Association between biochemical parameters and EVD. We investigated the association between biochemical parameters and EVD by comparing the values obtained within the first 2 days after admission in EVD patients and febrile controls (Figure 6A). AST and ALT levels were significantly higher in patients with EVD than in those in which EBOV infection was excluded. The concentrations of CPK, amylase, creatinine, and blood urea nitrogen were also significantly higher in EVD patients than in EBOV-negative patients. Higher CRP levels and lower $\mathrm{Ca}^{2+}$ concentrations were observed in EVD patients as opposed to EBOV-negative patients, but these differences were not significant. No significant difference was found between the different groups for levels of total bilirubin, albumin, glucose, $\mathrm{K}^{+}$, and $\mathrm{Na}^{+}$(data not shown). We observed a strong correlation between uremia and creatinine levels in EVD patients, whatever the outcome was (Figure 6B). Finally, significantly higher levels of IL-6 were detected at admission in fatal EVD cases as opposed to nonfatal EVD patients and EBOV-negative patients (Figure 6C). Furthermore, an IL-6 concentration over 29 pg/ml was significantly associated with a fatal outcome during EVD when a univariable analysis was performed (Table 1). However, this association was not significant with a multivariable analysis. 
A

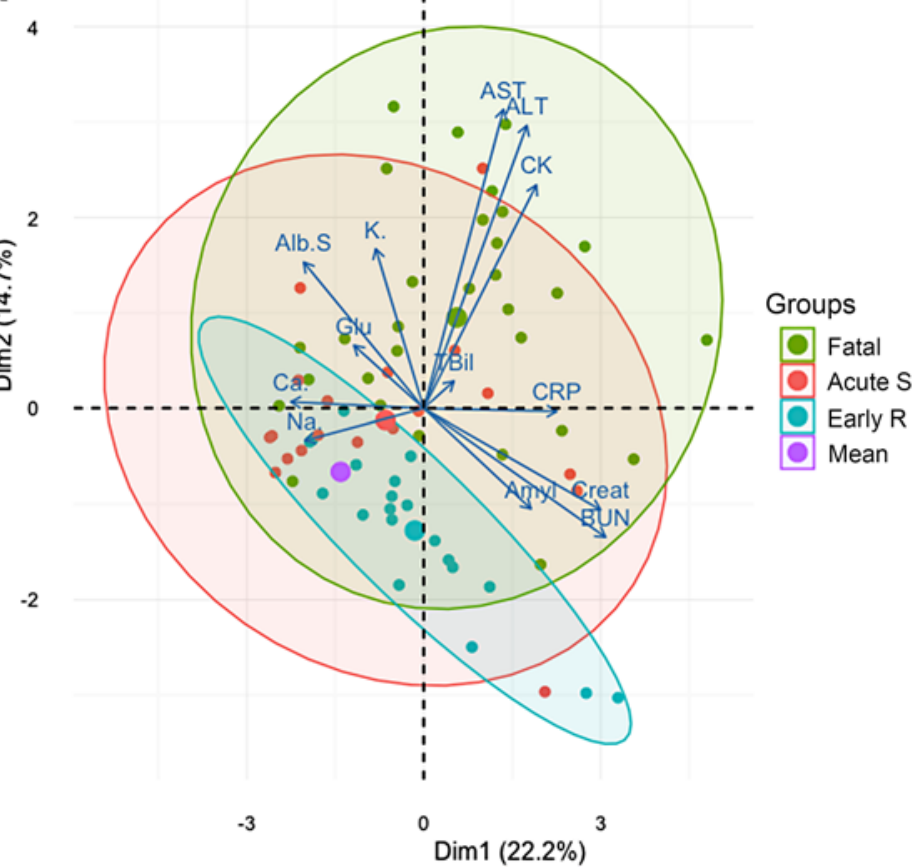

B
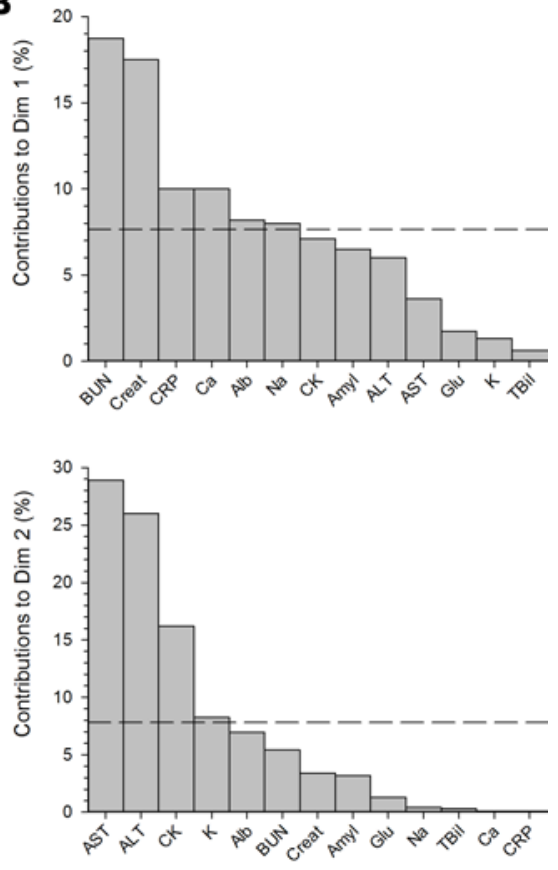

Figure 5. Principal component analysis of biological parameters throughout the course of the disease. Principal component analysis (PCA) of 13 different biological parameters in 3 distinct populations was performed. PCA is used to summarize the information contained in continuous multivariate data by reducing the dimensionality of the data without losing any important information. Samples obtained from surviving Ebola virus disease (EVD) patients during the symptomatic phase (i.e., 0-13 days after onset of symptoms) (Acute S) or during early recovery (more than 13 days after onset of symptoms) (Early R) and from fatal EVD patients (Fatal) were classified in 3 distinct groups on a 2-dimensional visualization plot of this PCA (A). The variables driving the transition between these populations, thus linked to prognosis, are represented by arrows. The purple circle represents the mean of the 3 populations. Contributions of these factors to dimensions 1 and 2 are represented in $\mathbf{B}$.

\section{Discussion}

Despite the limited period of time over which the ETC received patients and the laboratory processed samples, this study provides important insight into the changes in clinical, virological, and biological parameters during EVD. The outcome of EVD was independent of the time between symptom onset and admission, as observed at other ETCs $(5,9,10)$. Consistent with other reports $(9,10)$, the mortality rate was particularly high in patients under the age of 10 years or over the age of 45 years, but the differences were not significant. The mortality rate at our ETC was consistent with the global mortality recorded in Guinea (9). Almost all patients with $P$. falciparum and EBOV coinfection admitted to the ETC died, but this result was not significant. The high number of children below the age of 10 years among the $P$. falciparum-EBOV-positive patients (5 of 12 ) probably induced a bias in the mortality. Treatment for malaria (artemether-lumefantrine per os and artesunate i.v.) was systematically administered to all patients immediately on admission and was maintained when the patient tested positive for $P$. falciparum. We have no clear explanation for this observation of an aggravating effect of $P$. falciparum malaria. Plasmodium may bias the antiviral immune response, as this parasite is known to suppress adaptive immunity to heterologous antigens through the hemozoin-induced failure of dendritic cell functions (11). A role of the drug combination used at our ETC in the increased mortality is also a possibility, as suggested by a recent study showing a decrease in mortality in artesunate-amodiaquine-treated EVD patients compared with artesunate-lumefantrine-treated ones (12). Indeed, this treatment may be harmful because of the risk of prolongation of QT-interval and of fatal arrhythmias in patients presenting hypokalemia or hypomagnesemia. However, we were not able to evaluate the effect of artesunate-lumefantrine on mortality associated with prolonged QT-interval, as electrocardiograms were not available in the ETCs. The incidence of malaria was similar in all patients, regardless of EBOV infection status, and was particularly high. This incidence is consistent with the high rates of mortality unrelated to EVD observed in forested areas of Guinea during the outbreak, with most of these deaths due to untreated malaria and measles (13-15). In contrast, no increase in mortality was associated with coinfection with $P$. falciparum in another study in Guinea (16), and a recent report described a reverse trend and suggested that coinfection may 
A
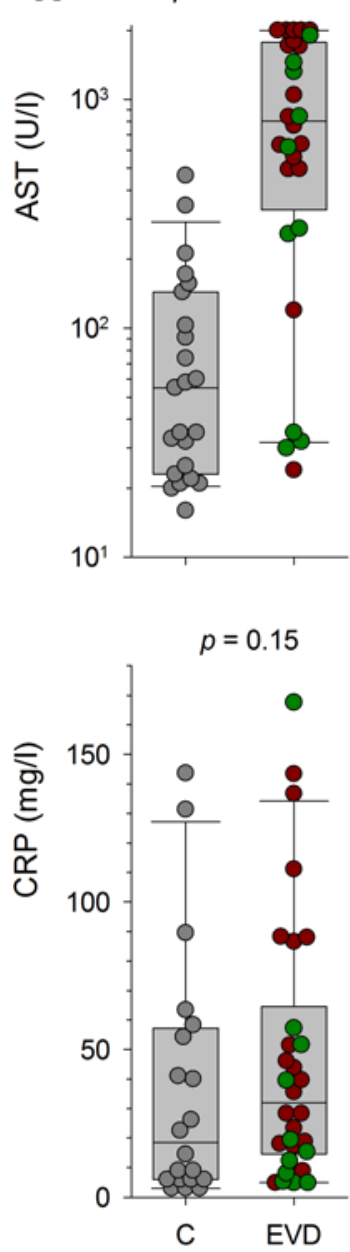

$p<0.001$

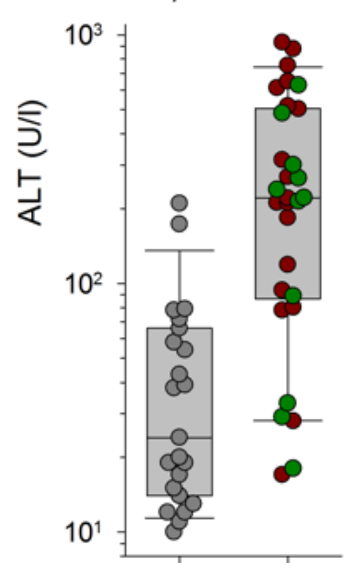

$10^{2}$

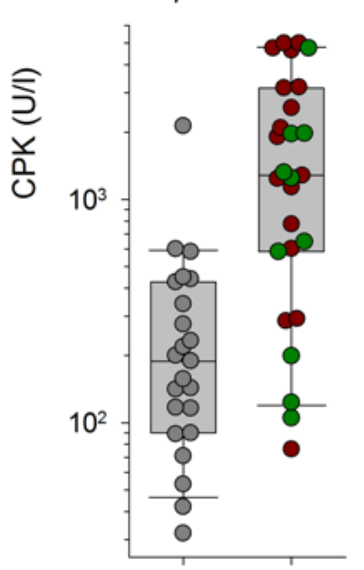

$p=0.031$

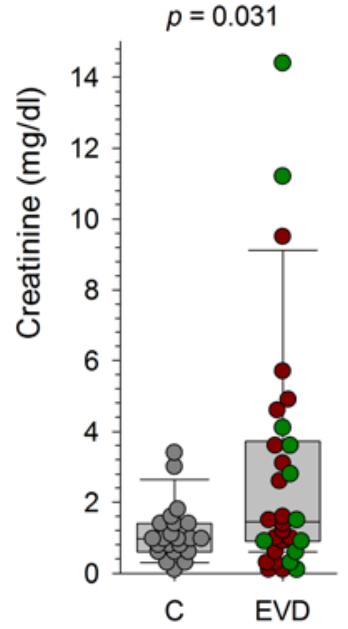

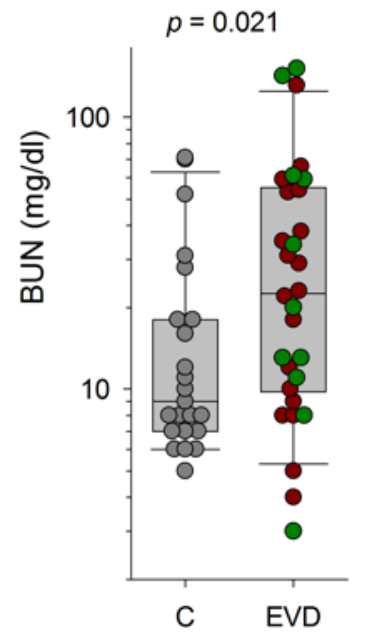

$p=0.007$
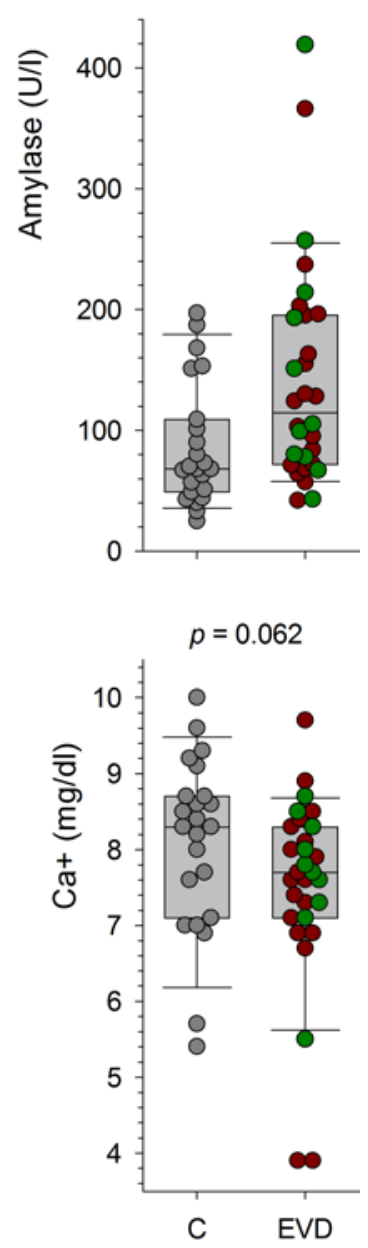
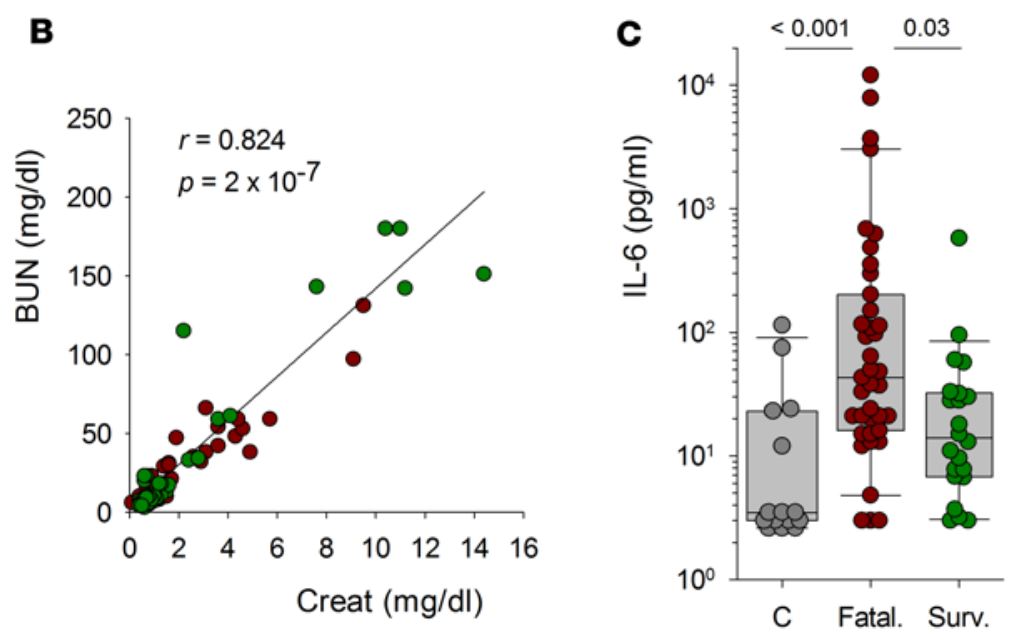

Figure 6. Association between biochemical parameters and EVD. (A) The levels of analytes measured within the first 2 days after admission are represented as individual values for febrile non-Ebola virus disease (non-EVD) patients (C, gray circles; $n=27$ ) and for patients with fatal (EVD, red circles; $n=27$ for aspartate aminotransferase [AST], creatine kinase [CPK], C-reactive protein [CRP], and corrected calcium [C ${ }^{2+}$ ]; $n=30$ for alanine aminotransferase [ALT], amylase, creatinine, and blood urea nitrogen [BUN]) and nonfatal (EVD, green circles; $n=19$ ) EVD. Box plots calculated using individual values from febrile controls ( $C ; n=27$ ) and from EVD patients (EVD; $n=46$ or 49) are also represented. The result of the Wilcoxon test used to compare the levels of analytes between febrile non-EVD patients and EVD patients is indicated for each analyte. (B) Correlation between BUN and creatinine levels in EVD patients $(n=68)$. Fatal (red circles, $n=30$ ) and nonfatal (green circles, $n=38$ ) patients are presented, but the statistical analysis was performed in all EVD patients. The regression line is shown, and the correlation coefficient and $P$ value calculated using a Spearman rank order correlation test are indicated. (C) Levels of IL-6 measured at admission are presented as individual values for febrile non-EVD patients (C, 
gray circles; $n=15$ ) and for patients with fatal (fatal., red circles; $n=39$ ) and nonfatal (Surv.; $n=22$ ) EVD. Box plots calculated using these individual values are also represented. The results of the ANOVA on ranks test used to compare the levels of IL- 6 between the different groups are indicated.

be associated with increased survival (17). Further investigation would therefore be needed to definitively clarify this point. Besides, the elevated number of $P$. falciparum-positive patients admitted in the ETC argues for a robust improvement in preventative measures against malaria in the general population to limit the admission of febrile EBOV-negative patients in the ETC.

Fever and/or asthenia were experienced by almost all EVD patients. Most symptoms, other than hemorrhagic symptoms and coma, occurred frequently during EVD and at the same frequency regardless of outcome. This finding contrasts with those of other reports, in which most other signs were more frequently observed in fatal cases than in survivors. This discrepancy probably reflects our reporting of the symptoms observed throughout the entire course of the disease, whereas other studies have focused on the clinical signs recorded at admission $(5,8,9,18,19)$. However, we have to keep in mind that the personal protective equipment used by the medical staff and the limited amount of time to examine the patients impeded the monitoring of clinical signs.

Viral load was high in all EBOV-infected patients, even those diagnosed 1 day after symptom onset. No initial negative EBOV RT-PCR results were obtained for symptomatic patients for whom positive results were obtained on subsequent days. There was just one dubious result for a patient admitted 2 days after symptom onset. However, given the very high viral load found the following day $(\mathrm{Ct}=16)$, this inconclusive result was probably due to technical problems with the RT-PCR assay rather than a low viral load. It is important to bear in mind that EBOV infection cannot be excluded on the basis of a negative EBOV RT-PCR result at early stages of the disease. Based on the results obtained, we decided to limit this early period of uncertainty to the first 3 days after the onset of EBOV-associated symptoms in a patient, rather than the 4-day waiting period that was initially used when the ETC first opened. Indeed, it is important to limit the time for which "negative" patients stay in the ETC to prevent their contamination with EBOV in the ward and the unnecessary occupation of beds and due to the lack of care appropriate for the patient's real illness. Viral load remained very high until death in fatal cases but declined rapidly about 1 week after onset in survivors, suggesting that immune responses were efficient at this time point. The difference in viral RNA load between outcomes was very large, and a viral load over 25,000 AI (arbitrary units) (corresponding to a $\mathrm{Ct}=19.5$ ) was significantly associated with fatal outcome. Thus, viral RNA load at admission was correlated with disease severity. Therefore, this parameter is of high prognostic value and could be used to select the patients in most need of intensive care or likely to benefit from experimental therapy. A similar conclusion was drawn in a study of the cohort recruited for the JIKI favipiravir trial and also in two cohorts in Sierra Leone $(20,21)$, demonstrating the robustness of this correlation. However, the difference between infectious viral load at admission according to the disease outcome was less pronounced than for RNA viral load and nonsignificant using a multivariate analysis, and this discrepancy remains unclear. We allowed the discharge of recovering patients after 2 negative EBOV RT-PCR tests, ideally performed 48 hours apart or 24 hours apart if the ETC was saturated. This interval between consecutive tests ensured that the patients discharged were aviremic and that viral load in urine had decreased considerably. It would be unrealistic and unfeasible to wait for the total disappearance of $E B O V$ RNA from the urine before discharging patients in the field in an epidemic setting. Moreover, as EBOV persists in semen for a very long time and as transmission of EBOV through sexual intercourse after recovery can occur (22-24), sexual advice and a hundred condoms were given to recovering patients before their discharge. Consistent with previous studies, which found a correlation between EVD severity and infectious viral load (25), we observed a similar trend in our patients, but the difference was not significant, probably because we did not quantify viral infectious load in patients with a low viral RNA load $(\mathrm{Ct}>27)$.

The acquisition of biological parameters during EVD provided us with useful data correlated with clinical observations, making it possible to optimize patient monitoring and care. In line with previous reports $(3-5,8,26,27)$, we confirmed that massive cytolysis occurred during EVD, particularly in patients with fatal infections who had high levels of AST and ALT. These data are consistent with hepatic damage, but the high AST/ALT ratio and CPK concentration also indicate that tissues other than the liver were harmed and that massive rhabdomyolysis was associated with the acute phase of EVD. Bilirubin levels were normal, suggesting that fulminant hepatic failure is not a feature of EVD, even in the most severe cases (5). The hypocalcemia observed in a substantial proportion of patients, which continued to worsen 
until death in fatal cases, is also consistent with rhabdomyolysis, which results in the transfer of free ionized $\mathrm{Ca}^{2+}$ into altered tissues (28). The mechanisms of acute virus-induced rhabdomyolysis are unknown, but these events can be linked to the intense myalgia frequently experienced by EBOV-infected patients (4) and may be involved in the pathogenic cascade that leads to terminal shock and death. Indeed, the necrosis of muscular tissue creates a "third space" in which intravascular fluid accumulates, leading to hypovolemia. In addition, muscle damage can lead to the release of endotoxins, vasoconstricting and prothrombotic compounds in the circulation, and therefore can participate in the shock syndrome, coagulation disorders, and renal failure observed during $\operatorname{EVD}(28,29)$. Levels of AST, and, to a lesser extent, ALT, appeared to be the most powerful markers of severity during EVD in our cohort. A similar observation was previously reported for EBOV and SUDV $(3-5,8)$. Striking differences were observed in the patterns of change in biological analyte levels associated with tissue damage during disease. Indeed, whereas AST, ALT, and CPK levels increased steadily until death in fatal cases, the opposite pattern was observed in survivors, with only a transient peak about 5 to 6 days after symptom onset, followed by a decline. These observations suggest that EBOV causes limited damage over a short time in nonfatal infections. A similar pattern was observed for IL-6, with high levels throughout the disease in fatal cases and only a moderate, transient increase in survivors. These findings differ from other studies of EBOV (30-32) in which concentrations of IL-6 were higher during nonfatal infections than in fatal cases, whereas consistent results were obtained with SUDV-infected patients (33) and with cynomolgus monkeys with severe Lassa fever (34). IL-6 is produced principally by activated monocytes/macrophages, mostly in liver. This release of IL-6 probably reflects tissue damage and hepatic regeneration (35) and could, therefore, be used as a marker of disease severity. High IL-6 levels may also result from tissue damage in other organs and muscles, consistent with the concomitantly high levels of AST. The high levels of IL-6 observed are also consistent with the fever associated with EVD, as this cytokine is pyrogenic (36). Moreover, IL-6 may be involved in pathogenesis, exacerbating endothelial permeability and fluid loss (37). High amylase concentrations were associated with EVD. Abnormally high amylase levels remained after the disappearance of viremia in survivors. These data may be consistent with acute pancreatitis, which could be linked to the intense epigastric and thoracic pain experienced by most patients, including two-thirds of survivors. Our findings differ from those reported for SUDV-infected patients, in whom high amylase levels were detected only in cases of fatal infection (3). Acute pancreatitis may also participate in hypocalcemia and more generally in the pathophysiological changes observed during EVD (38). However, multiple conditions other than pancreatitis are associated with high serum amylase, including salivary gland inflammation, renal failure, other acute abdominal syndromes, and tissue damages. As previously reported (5, 39), CRP concentrations increased in patients, indicating the occurrence of inflammatory responses during EVD. The persistence of abnormally high CRP levels after the disappearance of viremia in survivors is unclear. We hypothesize that this could be due to the occurrence of secondary bacterial infections in some patients because of bacterial translocation in the damaged intestinal tract (39), but further clinical investigation would be necessary to demonstrate it. Changes in renal function were also observed in some patients, particularly during severe disease, as illustrated by the high levels of creatinine and blood urea nitrogen and their highly significant correlation. However, unlike other studies $(4,5,8)$, we found that there was no significant correlation between high creatinine levels and a fatal outcome. Nevertheless, we found that the levels of both products increased during the course of the disease in fatal cases, suggesting that renal failure is a common feature of the terminal stages of EVD. Albumin concentration decreased throughout the disease in both groups of patients, with low levels persisting for up to 25 days after disease onset in survivors. This decrease may reflect undernourishment due to anorexia and vomiting, but it may also result from capillary leakage, third spacing, liver failure, and renal loss, as previously suggested (3). Hypoglycemia was also observed in some fatal cases, particularly in the last few days before death. With the exception of a small, nonsignificant decrease in hypoglycemia reported for SUDV-infected patients who died (3), we are aware of no other similar report. It is unclear whether hypoglycemia resulted from metabolic imbalance, undernutrition, and/or multiorgan failure, but it may have contributed to the fatal outcome, given the very low levels observed in the last 2 days before death in 4 patients. This hypoglycemia should be taken into account during rehydration. It would be interesting to investigate changes in glycemia during EVD further to determine its contribution to terminal shock and death. Electrolyte balance remained essentially in the normal range in most patients, except for a moderate decrease in $\mathrm{Ca}^{2+}$ levels relative to those in EBOV-negative patients. However, only some patients had values below the normal range. Hypocalcemia has already been described in patients that died from SUDV 
infection (3) and in some EBOV-infected patients (27). Kalaemia was normal in most patients, but hyperkalaemia was observed in $25 \%$ of fatal cases; a few fatal cases and survivors were hypokalemic, although hypokalemia was observed only after the acute phase of the disease in survivors. It is difficult to interpret these data, as there were probably antagonistic effects modulating $\mathrm{K}^{+}$levels: digestive loss due to vomiting and diarrhea, increases due to cell lysis, and input from Ringer lactate perfusion. However, the observation of hyperkalaemia only in fatal cases is consistent with a role of cell lysis in this event, as rhabdomyolysis leads to release of cellular $\mathrm{K}^{+}$into the circulation. Hyperkalaemia, together with hypocalcemia, can cause cardiac arrhythmias and even heart failure. It would be interesting to investigate the involvement of this phenomenon in the sudden death observed in some young patients in the ETC (28).

In conclusion, this study has increased our knowledge of the clinical, virological, and biological features associated with the different outcomes of EBOV infection. Our findings confirm that early viral load is a prognostic marker for the outcome and for the intensity of tissue damage. Rhabdomyolysis, liver damage, and inflammation were the main biological changes occurring during EVD, regardless of outcome. Our data suggest that coinfection with P. falciparum greatly affects mortality, despite treatment, but further investigations are required to confirm and explain this phenomenon. Finally, we confirmed that being able to perform biochemistry analyses within the ETC was important and of added value, making it possible to improve the supportive care tailored to the patient and to use the available human and drug resources rationally.

\section{Methods}

Study design. This prospective observational study included data for all suspected cases of EVD admitted to the Macenta or Nzerekore ETC between November 29, 2014, and January 31, 2015. The clinical data were collected from the patients' records at each ETC, and the biological data were obtained from The Pasteur Laboratory in Macenta. Patients with a confirmed diagnosis of EBOV infection were included and classified according to outcome. The patients for whom EBOV infection was excluded were included in the study as febrile controls. The number of blood samples obtained per patient was variable, ranging from a single sample for patients dying in the days following admission to 3-6 for survivors or patients for whom biochemical analyses were performed.

Blood samples. Blood samples were collected into $\mathrm{K}_{2}$ EDTA- or heparin-coated Vacutainer tubes (BD) for viral RT-PCR assays and biochemical analysis, respectively. All steps involving infectious biological samples were performed in a level-3 safety cabinet (Innosafe, Eurobioconcept). Patients were admitted to the ETC as EBOV-infected patients on the basis of a positive RT-PCR result for EBOV. If a negative result was obtained with a sample obtained fewer than 3 days after disease onset, another diagnostic test was performed on the following day. EBOV infection was excluded, and the patient was discharged from the ETC on the basis of a negative RT-PCR assay result for a sample obtained at least 3 days after the onset of symptoms. EBOV-infected patients were discharged once their symptoms had disappeared and 2 negative EBOV RT-PCR results had been obtained 48 hours apart.

Etiological diagnosis. Rapid tests for malaria (ONSITE PF/pan Malaria Ag Rapid Test, CTK Biotech) and typhoid fever (ONSITE Typhoid IgG/IgM Combo Rapid Test/K7, CTK Biotech) were performed with whole blood obtained at the admission of the patients. The malaria rapid test can discriminate between infections caused by $P$. falciparum and infections due to other species. Typhoid test results were considered positive only if IgM was detected.

EBOV infection was diagnosed by RT-PCR with the Real-Star Filovirus Screen RT-PCR kit 1.0 (Altona Diagnostics). Briefly, EDTA-treated blood was centrifuged, and total RNA was extracted from the resulting plasma with the QIAmp Viral RNA kit (Qiagen). RT-PCR was performed according to the manufacturer's specifications, with a Light Cycler 96 instrument (Roche Diagnostics). Positive results were reported in arbitrary units, calculated as follows: $2^{\Delta \text { cycle threshold }}$, with $\Delta \mathrm{Ct}=34$ - observed $\mathrm{Ct}$, assuming that $\mathrm{Ct}=34$ constituted the limit of sensitivity for the assay.

Care of patients. The patients included in this study have not received any EBOV-specific experimental therapy, and only supportive care was given to them. No advanced life support methods, such as artificial ventilation, dialysis, and insertion of central venous catheter, were available in the ETCs. Multivitamins, paracetamol (oral or parenteral), either an oral combination of artemether and lumefantrine or parenteral artesunate in case of severe vomiting and diarrhea, an empirical antimicrobial therapy (either oral ciprofloxacin or cefixime, or parenteral ceftriaxone), and anthelminthic albendazole were systematically administered to all patients whatever their EBOV status was. Tramadol, omeprazole, and diazepam were prescribed if needed. 
Oral rehydration salts and Plumpy'Nut (Nutriset) were given to all patients in addition to meals prepared twice a day. Ringer lactate was the main parenteral rehydration solution, with serum glucose $5 \%$ in addition sometimes. There was no specific care algorithm used to guide fluid and electrolyte therapy. The use of Ringer lactate solution and electrolyte therapy was limited to the most severely ill patients because a limited stock of parenteral rehydration solutions and electrolytes was available in the ETCs during the study period. When available, the results of biochemical analyses were used to select the patients receiving these therapies.

Biochemical analyses. Biochemical analyses were performed on whole blood with a Piccolo Xpress instrument and the Amlyte 13 panel (Abaxis), according to the manufacturer's recommendations. Corrected calcemia was calculated as follows: corrected $\left[\mathrm{Ca}^{2+}\right](\mathrm{mg} / \mathrm{dl})=$ total $\left[\mathrm{Ca}^{2+}\right](\mathrm{mg} / \mathrm{dl})+0.025 \times(40-[$ albumin $][$ in $\mathrm{g} / 1])$.

Virus titration. Vero E6 cells (ATCC) were incubated for 1 hour at $37^{\circ} \mathrm{C}$ with several dilutions of plasma, and 1.6\% carboxy-methyl-cellulose (BDH Laboratory Supplies) in DMEM (Life Technologies) was then added. Infectious foci were detected after 7 days of culture by incubation with a cocktail of 3 mouse monoclonal antibodies against EBOV GP, developed and provided by Laurent Bellanger (Laboratoire Innovations technologiques pour la Détection et le Diagnostic, Commissariat à l'énergie atomique, Marcoule, France) followed by alkaline phosphatase-conjugated goat polyclonal anti-mouse IgG (Sigma-Aldrich) and NBT/BCIP substrate (Thermo-Fisher). Results are expressed in FFU per ml.

IL-6 quantification. IL-6 was quantified in plasma from EBOV-infected patients $(n=99)$ and from febrile patients not infected with EBOV $(n=8)$ and in plasma from healthy donors $(n=6)$ with a Magpix instrument (EMD Millipore, Merck) and the Human Cytokine/Chemokine Magnetic Bead Panel (HCYTMAG60K-PX38, EMD Millipore, Merck), according to the manufacturer's instructions. Virus titration and IL-6 quantification were performed in the Jean Mérieux - INSERM BSL4 Laboratory.

Clinical data. Clinical data were collected from the patients' routine care records. These data, including the virological and biological data, were rendered anonymous and stored in a password-secured Microsoft Acces-based database.

Statistics. Univariate and multivariate logistical regressions were used to determine the associations between mortality during EVD and the following parameters at admission: age, sex, time between symptom onset and admission, viremia, infectious titers, IL-6, ETC, and presence of $P$. falciparum. A Wilcoxon test was used to compare levels of analytes between EBOV-negative patients and EVD patients in the first 2 days after admission, while ANOVA on ranks test was performed to compare the levels of IL- 6 at admission. Statistical analyses were performed with R 3.2.0 software and Sigma Plot 13.0 software (Systat Software). Principal component analysis was performed using the factoextra package under R software. $P$ values lower than 0.05 were considered significant.

Study approval. This study was approved by the Clinical Research Committee of Institut Pasteur (201516), the French Commission Nationale Informatique et Liberté (DR-2016-085; Paris, France), and the Guinean Comité National d'Ethique pour la Recherche en Santé (070/CNERS/15; Conakry, Guinea). As it has not been possible to obtain the written informed consent from patients, an exemption is included in the Guinean Comité National d'Ethique pour la Recherche en Santé agreement.

\section{Author contributions}

S Baize designed the study and drafted the manuscript. S Baize and MAV headed the Macenta laboratory during the study period. S Baize, SR, and AF designed and set up the Macenta laboratory. SR, AF, JS, DP, MM, PL, AP, and TMT performed diagnosis and analyses in the Macenta laboratory. MR, NG, NGB, AIS, PG, CS, SSC, MLDS, SH, BMLA, FP, NS, SS, MK, FRK, CL, and FLH collected clinical data and provided care to patients. CS and JYK headed the Macenta ETC. PZ and SL were in charge of the Macenta ETC pharmacy. LJ was in charge of health promotion and psychological care in the Nzerekore ETC. S Barbe coordinated the Ebola emergency response of Alliance for International Medical Action. BS, TTM, CL, and FLH provided medical expertise. S Baize, SR, and AP performed analyses in the INSERM Jean Mérieux BSL4 Laboratory. JG and MS performed statistical analyses. MAV, S Baize, SR, MR, MK, CL, and FLH analyzed data. All authors contributed to revising the manuscript.

\section{Acknowledgments}

This work was supported by funding from the French Ministry of Foreign Affairs, the Agence Française de Développement, and Institut Pasteur. We thank the Guinean Health Authorities in Conakry and Macenta and, particularly, S. Keita and L. Koivogui. We thank the French Embassy in Conakry for its help and, in 
particular, B. Cochery, R. Migliani, O. Buchbinder, R. Devouge, and P. Gibelot. We thank C. Taron-Brocard and C. Schmit (French Ministry of Foreign Affairs). We also thank C. Batejat (Environment and Infectious Risk Unit, Institut Pasteur) for training staff; M. Jouan, A. Granier-Blanc, K. Victoir, and M. Rabenoro (International Division, Institut Pasteur); and C. Rousseau (Purchase Department, Institut Pasteur). We are also particularly grateful to all the staff that worked in the Laboratoire Pasteur du CTE de Macenta from November 2014 to December 2015, including M. Poivogui in particular. We thank Z. Koivogui, M. Keita, S. Berete, C.M. Loua, R. Lolamou, A. Soumah, S. Condé, and M. Condé for their involvement in the collection of samples and data and care of patients. Finally, we thank the French Red Cross staff from Paris, Conakry, and Macenta for logistics.

Address correspondence to: Sylvain Baize, Unité de Biologie des Infections Virales Emergentes, Institut Pasteur, 21 avenue Tony Garnier, 69365 Lyon cedex 07, France. Phone 33.4.37.28.24.40; E-mail: sylvain.baize@pasteur.fr.

1. Baize S, et al. Emergence of Zaire Ebola virus disease in Guinea. N Engl J Med. 2014;371(15):1418-1425.

2. Baize S. Ebola virus in West Africa: new conquered territories and new risks-or how I learned to stop worrying and (not) love Ebola virus. Curr Opin Virol. 2015;10:70-76.

3. Rollin PE, Bausch DG, Sanchez A. Blood chemistry measurements and D-Dimer levels associated with fatal and nonfatal outcomes in humans infected with Sudan Ebola virus. J Infect Dis. 2007;196 Suppl 2:S364-S371.

4. Cournac JM, et al. Rhabdomyolysis in Ebola Virus Disease. Results of an Observational Study in a Treatment Center in Guinea. Clin Infect Dis. 2016;62(1):19-23.

5. Hunt L, et al. Clinical presentation, biochemical, and haematological parameters and their association with outcome in patients with Ebola virus disease: an observational cohort study. Lancet Infect Dis. 2015;15(11):1292-1299.

6. Schibler M, et al. Clinical features and viral kinetics in a rapidly cured patient with Ebola virus disease: a case report. Lancet Infect Dis. 2015;15(9):1034-1040.

7. Lyon GM, et al. Clinical care of two patients with Ebola virus disease in the United States. NEngl J Med. 2014;371(25):2402-2409.

8. Schieffelin JS, et al. Clinical illness and outcomes in patients with Ebola in Sierra Leone. N Engl J Med. 2014;371(22):2092-2100.

9. WHO Ebola Response Team. Ebola virus disease in West Africa--the first 9 months of the epidemic and forward projections. $N$ Engl J Med. 2014;371(16):1481-1495.

10. Bah EI, et al. Clinical presentation of patients with Ebola virus disease in Conakry, Guinea. N Engl J Med. 2015;372(1):40-47.

11. Millington OR, Di Lorenzo C, Phillips RS, Garside P, Brewer JM. Suppression of adaptive immunity to heterologous antigens during Plasmodium infection through hemozoin-induced failure of dendritic cell function. J Biol. 2006;5(2):5.

12. Gignoux E, et al. Effect of artesunate-amodiaquine on mortality related to Ebola virus disease. N Engl J Med. 2016;374(1):23-32.

13. Takahashi S, et al. Reduced vaccination and the risk of measles and other childhood infections post-Ebola. Science. 2015;347(6227):1240-1242.

14. Walker PG, White MT, Griffin JT, Reynolds A, Ferguson NM, Ghani AC. Malaria morbidity and mortality in Ebola-affected countries caused by decreased health-care capacity, and the potential effect of mitigation strategies: a modelling analysis. Lancet Infect Dis. 2015;15(7):825-832.

15. Plucinski MM, et al. Effect of the Ebola-virus-disease epidemic on malaria case management in Guinea, 2014: a cross-sectional survey of health facilities. Lancet Infect Dis. 2015;15(9):1017-1023.

16. Barry M, et al. Clinical predictors of mortality in patients with Ebola virus disease. Clin Infect Dis. 2015;60(12):1821-1824.

17. Rosenke K, et al. Plasmodium parasitemia associated with increased survival in Ebola virus-infected patients. Clin Infect Dis. 2016;63(8):1026-1033.

18. Li J, et al. Age and Ebola viral load correlate with mortality and survival time in 288 Ebola virus disease patients. Int J Infect Dis. 2016;42:34-39.

19. Lado M, et al. Clinical features of patients isolated for suspected Ebola virus disease at Connaught Hospital, Freetown, Sierra Leone: a retrospective cohort study. Lancet Infect Dis. 2015;15(9):1024-1033.

20. Sissoko D, et al. Experimental Treatment with Favipiravir for Ebola Virus Disease (the JIKI Trial): A Historically Controlled, Single-Arm Proof-of-Concept Trial in Guinea. PLoS Med. 2016;13(3):e1001967.

21. Lanini S, et al. Blood kinetics of Ebola virus in survivors and nonsurvivors. J Clin Invest. 2015;125(12):4692-4698.

22. Sow MS, et al. New evidence of long-lasting persistence of Ebola virus genetic material in semen of survivors. J Infect Dis. 2016;214(10):1475-1476.

23. Deen GF, et al. Ebola RNA Persistence in Semen of Ebola Virus Disease Survivors - Preliminary Report [published online ahead of print October 14, 2015]. N Engl J Med. https://doi.org/10.1056/NEJMoa1511410.

24. Mate SE, et al. Molecular evidence of sexual transmission of Ebola virus. N Engl J Med. 2015;373(25):2448-2454.

25. Ksiazek TG, et al. Clinical virology of Ebola hemorrhagic fever (EHF): virus, virus antigen, and IgG and IgM antibody findings among EHF patients in Kikwit, Democratic Republic of the Congo, 1995. J Infect Dis. 1999;179 Supp1 1:S177-S187.

26. Wolf T, et al. Severe Ebola virus disease with vascular leakage and multiorgan failure: treatment of a patient in intensive care. Lancet. 2015;385(9976):1428-1435.

27. Uyeki TM, et al. Clinical management of Ebola virus disease in the United States and Europe. N Engl J Med. 2016;374(7):636-646.

28. Chatzizisis YS, Misirli G, Hatzitolios AI, Giannoglou GD. The syndrome of rhabdomyolysis: complications and treatment. Eur J Intern Med. 2008;19(8):568-574.

29. Holt SG, Moore KP. Pathogenesis and treatment of renal dysfunction in rhabdomyolysis. Intensive Care Med. 2001;27(5):803-811. 
30. Baize S, et al. Inflammatory responses in Ebola virus-infected patients. Clin Exp Immunol. 2002;128(1):163-168.

31. Leroy EM, et al. Human asymptomatic Ebola infection and strong inflammatory response. Lancet. 2000;355(9222):2210-2215.

32. Villinger F, et al. Markedly elevated levels of interferon (IFN)-gamma, IFN-alpha, interleukin (IL)-2, IL-10, and tumor necrosis factor-alpha associated with fatal Ebola virus infection. J Infect Dis. 1999;179 Suppl 1:S188-S191.

33. Hutchinson KL, Rollin PE. Cytokine and chemokine expression in humans infected with Sudan Ebola virus. J Infect Dis. 2007;196 Supp1 2:S357-S363.

34. Baize S, et al. Early and strong immune responses are associated with control of viral replication and recovery in lassa virus-infected cynomolgus monkeys. J Virol. 2009;83(11):5890-5903.

35. Cressman DE, et al. Liver failure and defective hepatocyte regeneration in interleukin-6-deficient mice. Science. 1996;274(5291):1379-1383.

36. Dinarello CA. Infection, fever, and exogenous and endogenous pyrogens: some concepts have changed. J Endotoxin Res. 2004;10(4):201-222.

37. Maruo N, Morita I, Shirao M, Murota S. IL-6 increases endothelial permeability in vitro. Endocrinology. 1992;131(2):710-714.

38. Agarwal N, Pitchumoni CS. Acute pancreatitis: a multisystem disease. Gastroenterologist. 1993;1(2):115-128.

39. Kreuels B, et al. A case of severe Ebola virus infection complicated by gram-negative septicemia. N Engl J Med. 2014;371(25):2394-2401. 